Proceedings of the Edinburgh Mathematical Society (2002) 45, 353-362 (C)

DOI:10.1017/S0013091500001024 Printed in the United Kingdom

\title{
ADMISSIBLE AND WEAKLY ADMISSIBLE OBSERVATION OPERATORS FOR THE RIGHT SHIFT SEMIGROUP
}

\author{
BIRGIT JACOB ${ }^{1}$, JONATHAN R. PARTINGTON ${ }^{1}$ AND SANDRA POTT ${ }^{2}$ \\ ${ }^{1}$ School of Mathematics, University of Leeds, Leeds LS2 9JT, UK \\ (birgit@amsta.leeds.ac.uk; J.R.Partington@leeds.ac.uk) \\ ${ }^{2}$ Department of Mathematics, University of York, Heslington, \\ York YO10 5DD, UK (sp23@york.ac.uk)
}

(Received 12 October 2000)

\begin{abstract}
In this note we show that two conjectures of George Weiss on admissible and weakly admissible observation operators fail to hold in general for the right shift semigroup in the case that the output space is infinite dimensional.
\end{abstract}

Keywords: shift semigroup; admissibility; Hankel operator; observation operator

AMS 2000 Mathematics subject classification: Primary 47D06; 32A37; 93B28

\section{Introduction}

In this paper we are concerned with linear systems of the following kind:

$$
\left.\begin{array}{l}
\dot{x}(t)=A x(t), \\
y(t)=C x(t),
\end{array}\right\} \quad t \geqslant 0 .
$$

Here $x(t) \in H$, where $H$ is a Hilbert space, is the state of the system at time $t \geqslant 0$ and $y \in L^{2}(0, \infty ; Y)$ is the output of the system. $Y$ is another Hilbert space. The space $H$ is called the state space, and the space $Y$ is called the output space. In (1.1), both $A$ and $C$ are possibly unbounded operators. $A$ is the infinitesimal generator of a $C_{0}$-semigroup $T(t)$ on $H$ and $C$ is assumed to be a linear bounded operator from $D(A)$, the domain of $A$, to $Y$. However, in general $C$ will not be a bounded operator from $H$ to $Y$. By a solution of $\dot{x}(t)=A x(t)$ with initial condition $x(0)=x_{0} \in H$ we mean the continuous function

$$
x(t)=T(t) x_{0}, \quad t \geqslant 0 .
$$

These assumptions are not sufficient to guarantee that the output of the system is in $L^{2}(0, \infty ; Y)$. In order to guarantee this an additional assumption is needed. Following Weiss [12] we introduce admissible observation operators for $T(t)$. Let $X$ and $Y$ be normed spaces; by $\mathcal{L}(X, Y)$ we denote the set of bounded linear operators from $X$ to $Y$. 
Definition 1.1. Let $C \in \mathcal{L}(D(A), Y)$. Then $C$ is called an (infinite-time) admissible observation operator for $T(t)$, if there is some $K>0$ such that

$$
\|C T(\cdot) x\|_{L^{2}(0, \infty ; Y)} \leqslant K\|x\|, \quad x \in D(A) .
$$

Let $C \in \mathcal{L}(D(A), Y)$. Then it is easy to show that Condition 1 implies Condition 2, where

1. $C$ is an infinite-time admissible observation operator for $T(t)$;

2. for every $\phi \in Y^{\prime}$ we have that $\phi C$ is an infinite-time admissible observation operator for $T(t)$.

We refer to Condition 2 as weak admissibility. Clearly, it is the same as admissibility if $Y$ is finite dimensional.

Weiss [13] conjectured that Condition 2 implies Condition 1, and he proved this implication in that case that $T(t)$ is merely right invertible and exponentially stable. Moreover, in Hansen and Weiss [6] it is shown that Condition 2 implies Condition 1 if $T(t)$ is exponentially stable, normal and analytic. Further technical results concerning the implication from Condition 2 to Condition 1 can be found in Hansen and Weiss [7]. Quite recently Zwart, Jacob and Staffans [15] constructed an example showing that this implication does not hold in general. In their example the operators $A$ and $C$ are bounded and $T(t)$ generates a strongly stable semigroup.

In this note we present another example showing that this implication is in general not true. The $C_{0}$-semigroup we choose in our example is even a contraction semigroup, namely, the right shift on $H^{2}\left(\mathbb{C}_{+}, H\right)$, where $H$ is an infinite-dimensional Hilbert space. Infinite-time admissibility also implies the following resolvent condition.

3. There exists a constant $m>0$ such that

$$
\left\|C(s I-A)^{-1}\right\| \leqslant \frac{m}{\sqrt{\operatorname{Re} s}}, \quad s \in \mathbb{C}_{+} .
$$

Our example shows that Condition 3 is not sufficient for the admissibility of $C$, that is, Condition 3 does in general not imply Condition 1 even if we restrict ourselves to contraction semigroups. This answers another question posed by Weiss [13], see also Weiss [14]. Note that Weiss showed that Condition 3 implies Condition 1 if $T(t)$ is exponentially stable and right invertible. Moreover, Partington and Weiss [11] showed that this implication holds for the right shift on $H^{2}\left(\mathbb{C}_{+}\right)$and finite-dimensional output spaces, and in Jacob and Partington [8] their techniques were generalized to contraction semigroups using the Sz.-Nagy-Foiaş model theory. Again, Zwart, Jacob and Staffans [15] showed that this implication even fails if we restrict ourselves to bounded operators $A$ and $C$, and quite recently Jacob and Zwart [9] showed that this implication does not hold for analytic $C_{0}$-semigroups in the case that $Y$ is finite dimensional. 


\section{Presentation of the example}

Throughout this section we assume the following: $H$ is an infinite-dimensional Hilbert space,

$$
\begin{aligned}
Z & :=H^{2}\left(\mathbb{C}_{+}, H\right), \\
(A x)(s) & :=-s x(s), \quad x \in D(A), \quad s \in \mathbb{C}_{+}, \\
D(A) & :=\{x \in Z \mid \mathrm{i} \omega x(\mathrm{i} \omega) \in Z\}, \\
(T(t) x)(s) & :=\mathrm{e}^{-s t} x(s), \quad t \geqslant 0, \quad x \in Z, \quad s \in \mathbb{C}_{+} .
\end{aligned}
$$

$T(t)$ is the right shift semigroup on $Z$ with infinitesimal generator $A$. Here $\mathbb{C}_{+}$denotes the right half-plane $\{s \in \mathbb{C} \mid \operatorname{Re} s>0\}$, and $H^{2}\left(\mathbb{C}_{+}, H\right)$ denotes the Hardy space of $H$ valued functions on the right half-plane, which is a closed subspace of $L^{2}(\mathrm{i} \mathbb{R}, H)$. By $\mathbb{C}_{-}$ we denote the set $\{s \in \mathbb{C} \mid \operatorname{Re} s<0\}$, and $H^{2}\left(\mathbb{C}_{-}, H\right)$ is the corresponding Hardy space. Moreover, $P_{+}$denotes the orthogonal projection from $L^{2}(i \mathbb{R}, H)$ onto $H^{2}\left(\mathbb{C}_{+}, H\right), P_{-}$ denotes the orthogonal projection onto $H^{2}\left(\mathbb{C}_{-}, H\right)$, and $J: H^{2}\left(\mathbb{C}_{+}, H\right) \rightarrow H^{2}\left(\mathbb{C}_{-}, H\right)$ is given by

$$
(J x)(s):=x(-s) .
$$

We will freely identify any locally integrable scalar- or vector-valued function $f$ on $i \mathbb{R}$ with its Poisson extension to the right (respectively, left) half-plane (provided it exists), and we will denote the Poisson extension in $z \in \mathbb{C}_{+}$(respectively, $\mathbb{C}_{-}$) by $f(z)$. We call $f$ analytic, if its Poisson extension to $\mathbb{C}_{+}$is analytic.

The following theorem will be crucial.

Theorem 2.1. There exists an analytic operator-valued function $B: \mathrm{i} \mathbb{R} \rightarrow \mathcal{L}(H)$ such that

(i) $B(s)^{*} h /(1+s) \in L^{2}(\mathrm{i} \mathbb{R}, H)$ for every $h \in H$, and there exists a constant $M>0$ such that $\left\|B(s)^{*} h /(1+s)\right\|_{L^{2}(\mathbb{R}, H)} \leqslant M\|h\|$ for all $h \in H$;

(ii) the Hankel operator

$$
\Gamma_{B}:\left\{x \in H^{2}\left(\mathbb{C}_{+}, H\right) \mid B(J x) \in L^{2}(\mathrm{i} \mathbb{R}, H)\right\} \rightarrow H^{2}\left(\mathbb{C}_{+}, H\right)
$$

defined by

$$
\Gamma_{B} x:=P_{+}(B(J x)), \quad x \in H^{2}\left(\mathbb{C}_{+}, H\right),
$$

cannot be extended to a bounded operator on $H^{2}\left(\mathbb{C}_{+}, H\right)$;

(iii) there exists a constant $m>0$ such that

$$
\left\|\left(\Gamma_{B}\right)^{*}\left(f_{z} h\right)\right\| \leqslant m\|h\|, \quad \text { for every } z \in \mathbb{C}_{+}, \quad h \in H,
$$

where $f_{z} \in H^{2}\left(\mathbb{C}_{+}\right), z \in \mathbb{C}_{+}$, is given by

$$
f_{z}(s):=\frac{\sqrt{\operatorname{Re} z}}{s+z}, \quad z, s \in \mathbb{C}_{+} .
$$


Remarks. This can be formulated as saying that Bonsall's Theorem is not valid in infinite dimensions, in the sense that the boundedness of the adjoint Hankel operator $\left(\Gamma_{B}\right)^{*}$ (or equivalently, the boundedness of the Hankel operator $\Gamma_{\tilde{B}}$ with operator symbol $\left.\tilde{B}(s)=B^{*}(-s)\right)$ on the normalized Szegö kernels $f_{z} h, z \in \mathbb{C}_{+}, h \in H,\|h\|=1$ does not imply the boundedness of $\left(\Gamma_{B}\right)^{*}$ (or $\Gamma_{\tilde{B}}$ ).

A stronger result has been proved in [5] for the case of the unit circle: even the boundedness of both $\Gamma_{B}$ and $\left(\Gamma_{B}\right)^{*}$ on the normalized Szegö kernels does not imply the boundedness of $\Gamma_{B}$. It is also not difficult to derive a counterexample from the 'dyadic case' treated in $[\mathbf{1 0}]$.

However, it is easier to show that boundedness of $\Gamma_{B}$ and $\left(\Gamma_{B}\right)^{*}$ on the normalized Szegö kernels are not equivalent. We will do that here, presenting an elementary selfcontained counterexample. The boundedness of $\Gamma_{B}$ and of $\left(\Gamma_{B}\right)^{*}$ on the normalized Szegö kernels have interpretations as natural BMO conditions on the operator function $B$ (see, for example, [5]).

It remains to ask whether, similar to the boundedness of $\left(\Gamma_{B}\right)^{*}$ on the normalized Szegö kernels reflecting weak admissibility, the boundedness of $\Gamma_{B}$ on the normalized Szegö kernels also reflects an interesting property of the associated linear system, and whether the above-mentioned counterexample from [5] therefore contains interesting information about linear systems.

Proof of Theorem 2.1. We consider operator functions of the form $B=b \otimes \bar{e}$, where $b$ is a locally integrable $H$-valued function on $i \mathbb{R}, e$ is a fixed unit vector in $H$, and the bar stands for componentwise complex conjugation (relative to some fixed orthonormal basis of $H$ ). By an imitation of the well-known calculation of Bonsall (see [3], p. 290-291, eqns (1)-(5)), we have

$$
\begin{aligned}
\left\|\left(\Gamma_{B}\right)^{*} f_{z} h\right\|^{2} & =\left\langle B B^{*}(z) h, h\right\rangle-\left\langle B(z) B^{*}(z) h, h\right\rangle \\
& =\|\langle b, h\rangle\|^{2}(z)-\|\langle b(z), h\rangle\|^{2} \quad \text { for } h \in H, \quad\|h\|=1, \quad z \in \mathbb{C}_{+} .
\end{aligned}
$$

Taking a fixed $h \in H$, the supremum of the above expression over all $z \in \mathbb{C}_{+}$is just (up to equivalence) the square of the BMO-norm of the scalar-valued function $\langle b, h\rangle$ (see, for example, [4], p. 234, Corollary VI.2.4).

So asking for uniform boundedness of expression (2.1) means that we require the vector-valued function $b$ to be weakly BMO in the sense that $\langle b, h\rangle$ is in scalar-valued BMO uniformly for all $h \in H,\|h\|=1$. We call the space of those functions $\operatorname{WBMO}(H)$ and equip it with the norm

$$
\sup _{\substack{h \in H,\|h\|=1}} \sup _{I \subset \mathbb{R} \text { interval }} \frac{1}{|I|}\left(\int_{I}\left|\langle b(t), h\rangle-\left\langle m_{I} b, h\right\rangle\right|^{2} \mathrm{~d} t\right)^{1 / 2},
$$

which is equivalent to the square root of the supremum of expressions in (2.1).

On the other hand,

$$
\sup _{\substack{h \in H,\|h\|=1}}\left\|\Gamma_{B} f_{z} h\right\|^{2}=\sup _{\substack{h \in H,\|h\|=1}}\left\langle B^{*} B(z) h, h\right\rangle-\left\langle B(z)^{*} B(z) h, h\right\rangle
$$




$$
\begin{aligned}
& =\sup _{\substack{h \in H \\
\|h\|=1}}\|b\|^{2}(z)|\langle e, h\rangle|^{2}-\|b(z)\langle e, h\rangle\|^{2} \\
& =\|b\|^{2}(z)-\|b(z)\|^{2} \quad \text { for } z \in \mathbb{C}_{+} .
\end{aligned}
$$

The supremum over all $z \in \mathbb{C}_{+}$is equivalent to the (strong) vector BMO norm of $b$, namely

$$
\sup _{I \subset \mathrm{iR} \text { interval }} \frac{1}{|I|}\left(\int_{I}\left\|b(t)-m_{I} b\right\|^{2} \mathrm{~d} t\right)^{1 / 2} .
$$

We denote the space of all $H$-valued functions for which this expression is finite by $\operatorname{BMO}(H)$.

We now compare the weak and the strong BMO norm of $H$-valued functions in the above sense. The following lemma seems to be known, although the authors are not aware of it appearing in the literature. Note that, by taking an orthonormal basis of $H$, we may (for each $N \geqslant 1$ ) regard $\mathbb{C}^{N}$ as being isometrically embedded as a subspace of $H$.

Lemma 2.2. Let $H$ be an infinite-dimensional separable Hilbert space. Then

$$
\operatorname{BMO}(H) \subsetneq \operatorname{WBMO}(H)
$$

Moreover, there exists a constant $c>0$ such that for each positive integer $N$, there exists a $\mathbb{C}^{N}$-valued function $b_{N}$ on $i \mathbb{R}$ with

$$
\left\|b_{N}\right\|_{\text {WBmo }(H)} \leqslant c \frac{1}{N^{1 / 2}}\left\|b_{N}\right\|_{\operatorname{BMo}(H)} .
$$

Proof. Let $\mathcal{D}$ denote the standard system of dyadic subintervals of $\mathbb{R}$, with $[0,1] \in \mathcal{D}$. For $I, J \in \mathcal{D}, I \subsetneq J$, let $\operatorname{sgn}(I, J)=1$, if $I$ is contained in the left half of $J$, and $\operatorname{sgn}(I, J)=-1$, if $I$ is contained in the right half of $J$. Similarly, for each $I \in \mathcal{D}$, let $\operatorname{sgn}(I)$ equal 1 (respectively, -1 ) if $I$ is contained in the left (respectively, right) half of its dyadic mother interval.

Let $e_{1}, \ldots, e_{N}$ denote the standard basis of $\mathbb{C}^{N}$, and let $e_{I}:=e_{k}$ for $|I|=2^{-k}$ and $e_{I}=0$ for $|I| \geqslant 1$ or $|I|<2^{-N}$. For $I \in \mathcal{D}$, define $b_{I}=\operatorname{sgn}(I)|I|^{1 / 2} e_{I}$.

We now define $b$ by

$$
b(\mathrm{i} t)=\sum_{I \in \mathcal{D}} b_{I} h_{I}(t)
$$

where $h_{I}$ denotes the Haar function $|I|^{-1 / 2}\left(\chi_{I^{+}}-\chi_{I^{-}}\right)$and $I^{+}$(respectively, $\left.I^{-}\right)$denotes the left (respectively, right) half of $I$.

We obtain

$$
\int_{[0,1]}\left\|b(\mathrm{i} t)-m_{\mathrm{i}[0,1]} b\right\|^{2} \mathrm{~d} t=\sum_{\substack{I \in \mathcal{D}, I \subsetneq[0,1]}}\left\|b_{I}\right\|^{2}=\sum_{\substack{I \in \mathcal{D}, I \subsetneq[0,1], 2^{-N} \leqslant|I|<1}}|I|\left\|e_{I}\right\|^{2}=N
$$

thus $\|b\|_{\mathrm{BMO}(H)} \geqslant N^{1 / 2}$. 
On the other hand, for any $J \in \mathcal{D}$ and $h \in H$ with $\|h\|=1$, we have

$$
\begin{aligned}
\int_{J}\left|\left\langle b(\mathrm{i} t)-m_{\mathrm{i} J} b, h\right\rangle\right|^{2} \mathrm{~d} t & =\sum_{I \subsetneq J}\left|\left\langle b_{I}, h\right\rangle\right|^{2} \\
& =\sum_{I \subsetneq J}\left\|b_{I}\right\|^{2}\left|\left\langle e_{I}, h\right\rangle\right|^{2}=\sum_{I \subsetneq J}|I|\left|\left\langle e_{I}, h\right\rangle\right|^{2} \leqslant|J| .
\end{aligned}
$$

To check the WBMO condition for $b$, it is now sufficient to compare $\left\langle m_{\mathrm{i} I} b, h\right\rangle$ and $\left\langle m_{\mathrm{i} I^{\prime}} b, h\right\rangle$ for neighbouring dyadic intervals $I, I^{\prime}$ of the same length (see [4], p. 274).

Let $\tilde{I}$ be the smallest dyadic interval containing both $I$ and $I^{\prime}$. Then

$$
\begin{aligned}
\left\langle m_{\mathrm{i} I} b, h\right\rangle & -\left\langle m_{\mathrm{i} I^{\prime}} b, h\right\rangle \\
& =\sum_{J \in \mathcal{D}, J \supsetneq I} \operatorname{sgn}(I, J) \frac{1}{|J|^{1 / 2}}\left\langle b_{J}, h\right\rangle-\sum_{J^{\prime} \in \mathcal{D}, J^{\prime} \supsetneq I^{\prime}} \operatorname{sgn}\left(I^{\prime}, J^{\prime}\right) \frac{1}{\left|J^{\prime}\right|^{1 / 2}}\left\langle b_{J^{\prime}}, h\right\rangle .
\end{aligned}
$$

It is not difficult to see that the contributions from all $J, J^{\prime}$ in the above sum apart from $J=J^{\prime}=\tilde{I}$ cancel out, and we are left with

$$
2 \operatorname{sgn}(I, \tilde{I}) \frac{1}{|\tilde{I}|^{1 / 2}}\left\langle b_{\tilde{I}}, h\right\rangle .
$$

Thus $\left|\left\langle m_{\mathrm{i} I} b, h\right\rangle-\left\langle m_{\mathrm{i} I}{ }^{\prime} b, e\right\rangle\right| \leqslant 2$. Using a standard argument about the comparison of $\mathrm{BMO}$ and dyadic BMO (see, for example, $[4]$, p. 274 again), we obtain that $\|b\|_{\mathrm{WBMO}(H)} \leqslant$ $c$ with a constant $c>0$ independent of $N$. Setting $b_{N}=b$ finishes the proof of the lemma.

Note that also

$$
\left\|\frac{1}{1+t}\left\langle b_{N}, h\right\rangle\right\|_{L^{2}(\mathrm{i} \mathbb{R}, \mathrm{d} t)} \leqslant\left(2+2 \sum_{n=1}^{\infty} \frac{1}{n^{2}}\right)\left\|\left\langle b_{N}, h\right\rangle\right\|_{L^{2}(\mathrm{i}[0,1], \mathrm{d} t)} \leqslant\left(2+2 \sum_{n=1}^{\infty} \frac{1}{n^{2}}\right)
$$

for all $h \in H,\|h\|=1$ and all positive integers $N$. But

$$
\left\|\frac{\left(b_{N} \otimes \bar{e}\right)^{*}}{1+t} h\right\|_{L^{2}(\mathbb{R} R, \mathrm{~d} t)}=\left\|\frac{1}{1+t}\left\langle b_{N}, h\right\rangle\right\|_{L^{2}(\mathbb{R} R, \mathrm{~d} t)},
$$

which gives the estimate in property (i) of Theorem 2.1.

We still require $B$ to be analytic, i.e. $b$ has to be analytic. But since the Riesz projection $P_{+}: L^{2}(\mathrm{iR}, H) \rightarrow H^{2}\left(\mathbb{C}_{+}, H\right)$ extends to a bounded linear operator on both WBMO $(H)$ and $\mathrm{BMO}(H)$ (this follows from the boundedness of the Hilbert transform on scalar BMO and also on $\operatorname{BMO}(H)$; see, for example, [2]), we have

$$
c_{1}\|b\|_{\mathrm{WBMO}(H)} \leqslant\left\|P_{+} b\right\|_{\mathrm{WBMO}(H)}+\left\|P_{-} b\right\|_{\mathrm{WBMO}(H)} \leqslant c_{2}\|b\|_{\mathrm{WBMO}(H)}
$$

and

$$
c_{1}\|b\|_{\mathrm{BMO}(H)} \leqslant\left\|P_{+} b\right\|_{\mathrm{BMO}(H)}+\left\|P_{-} b\right\|_{\mathrm{BMO}(H)} \leqslant c_{2}\|b\|_{\mathrm{BMO}(H)}
$$


with constants $c_{1}, c_{2}>0$ independent of $b$ and $N$. So, replacing each $b_{N}$ in the lemma above by either $P_{+} b_{N}$ or $J P_{-} b_{N}$, we obtain the statement of the lemma also for analytic $\mathbb{C}^{N}$-valued functions. These modified functions still satisfy estimates (2.3) and (2.4) (up to a constant) and equation (2.5).

Altogether, we have the following.

Lemma 2.3. There exist constants $M, m, c>0$ such that for each positive integer $N$, there is an analytic function $b_{N}: \mathrm{i} \mathbb{R} \rightarrow \mathbb{C}^{N}$ with

(i) $\left\|b_{N}\right\|_{\mathrm{WBMO}(H)} \leqslant m$,

(ii) $\left\|b_{N}\right\|_{\mathrm{BMO}(H)} \geqslant N^{1 / 2} / c$,

(iii) $\left|\left\langle\frac{1}{1+s} b_{N}, h\right\rangle\right|_{L^{2}(\mathrm{i} \mathbb{R})} \leqslant M$ for all $h \in H,\|h\|=1$.

In particular, $\left\|\left(b_{N}(s) \otimes \bar{e}\right)^{*} h /(1+s)\right\|_{L^{2}(\mathbb{R}, H)} \leqslant M$ for $h \in H,\|h\|=1$.

Letting $N \rightarrow \infty$ and using the Banach Open Mapping Theorem now finishes the proof of Theorem 2.1.

We can now prove the main result of this paper.

Theorem 2.4. There exists an operator $C: D(A) \rightarrow H$ such that

(i) $C \in \mathcal{L}(D(A), H)$;

(ii) $C$ is not an admissible observation operator for $T(t)$;

(iii) there exists a constant $m>0$ such that

$$
\left\|C(z I-A)^{-1}\right\| \leqslant \frac{m}{\sqrt{\operatorname{Re} z}} \quad\left(z \in \mathbb{C}_{+}\right)
$$

(iv) for every $\phi \in H^{\prime}$ we have that $\phi C$ is an admissible observation operator, that is, $C$ is weakly admissible.

Proof. We define the operator $C: D(A) \rightarrow H$ by

$$
\langle C x, h\rangle=\int_{-\infty}^{\infty}\langle B(-\mathrm{i} \omega) x(\mathrm{i} \omega), h\rangle \mathrm{d} \omega, \quad x \in D(A), \quad h \in H
$$

where $B$ is as in Theorem 2.1. Then $C$ satisfies properties (i)-(iv) as follows. 
(i) Let $x \in D(A), h \in H,\|h\|=1$, and define $x_{0} \in H^{2}\left(\mathbb{C}_{+}, H\right)$ by $x_{0}=(I-A) x$. Then we have

$$
\begin{aligned}
|\langle C x, h\rangle|^{2} & =\left|\int_{-\infty}^{\infty}\left\langle B(-\mathrm{i} \omega)(I-A)^{-1} x_{0}(\mathrm{i} \omega), h\right\rangle \mathrm{d} \omega\right|^{2} \\
& =\left|\int_{-\infty}^{\infty}\left\langle\frac{B(-\mathrm{i} \omega)}{1+\mathrm{i} \omega} x_{0}(\mathrm{i} \omega), h\right\rangle \mathrm{d} \omega\right|^{2} \\
& =\left|\int_{-\infty}^{\infty}\left\langle x_{0}(\mathrm{i} \omega), \frac{B^{*}(-\mathrm{i} \omega)}{1-\mathrm{i} \omega} h\right\rangle \mathrm{d} \omega\right|^{2} \\
& \leqslant \int_{-\infty}^{\infty}\left\|x_{0}(\mathrm{i} \omega)\right\|^{2} \mathrm{~d} \omega \int_{-\infty}^{\infty}\left\|\frac{B^{*}(-\mathrm{i} \omega)}{1-\mathrm{i} \omega} h\right\|^{2} \mathrm{~d} \omega \\
& \leqslant M\left\|x_{0}\right\|^{2} \leqslant M(\|x\|+\|A x\|)^{2},
\end{aligned}
$$

where $M>0$ is independent of $x \in D(A)$.

(ii) Since the Hankel operator $\Gamma_{B}$ in Theorem 2.1 has no bounded extension to $H^{2}\left(\mathbb{C}_{+}, H\right)$, we see that, given any $M>0$, there is an $x \in H^{2}\left(\mathbb{C}_{+}, H\right)$ of norm 1 such that $B(J x) \in L^{2}(\mathrm{i} \mathbb{R}, H)$ and $\left\|P_{+} B(J x)\right\|>M$. By considering the function $s \mapsto x(s) /(1+\epsilon s)$ for sufficiently small $\epsilon>0$, we may assume in addition that $(1+s) x(s)$ and $(1+s) B(J x)(s)$ both lie in $L^{2}(\mathrm{i} \mathbb{R}, H)$. In particular, $x \in D(A)$ and $B(J x) \in L^{1}(\mathbb{i} \mathbb{R}, H)$.

Now for any $t \geqslant 0$ and any $h \in H$, we obtain

$$
\begin{aligned}
\langle C T(t) x, h\rangle & =\int_{-\infty}^{\infty}\left\langle B(-\mathrm{i} \omega)\left(\mathrm{e}^{-\mathrm{i} \omega t} x(\mathrm{i} \omega)\right), h\right\rangle \mathrm{d} \omega \\
& =\int_{-\infty}^{\infty}\left\langle\mathrm{e}^{-\mathrm{i} \omega t} B(-\mathrm{i} \omega) x(\mathrm{i} \omega), h\right\rangle \mathrm{d} \omega \\
& =\int_{-\infty}^{\infty}\left\langle\mathrm{e}^{\mathrm{i} \omega t} B(\mathrm{i} \omega) x(-\mathrm{i} \omega), h\right\rangle \mathrm{d} \omega \\
& =2 \pi\left\langle\mathcal{L}_{\mathrm{b}}^{-1}(B(J x))(t), h\right\rangle,
\end{aligned}
$$

where $\mathcal{L}_{\mathrm{b}}: L^{2}(\mathbb{R}, H) \rightarrow L^{2}(\mathrm{i} \mathbb{R}, H)$ denotes the bilateral Laplace transform. That is, $C T(t) x$ is the inverse Laplace transform of $2 \pi P_{+}(B(J x))$. Thus we get

$$
\left(\int_{0}^{\infty}\|C T(t) x\|^{2} \mathrm{~d} t\right)^{1 / 2}=2 \pi\left\|P_{+}(B(J x))\right\| \geqslant M 2 \pi\|x\|,
$$

which proves that $C$ is not an admissible observation operator for $T(t)$. 
(iii) For $z \in \mathbb{C}_{+}, x \in H^{2}\left(\mathbb{C}_{+}, H\right)$ and $h \in H$ we have

$$
\begin{aligned}
\left\langle h, C(z I-A)^{-1} x\right\rangle & =\int_{-\infty}^{\infty}\left\langle h, B(-\mathrm{i} \omega) \frac{x(\mathrm{i} \omega)}{z+\mathrm{i} \omega} \mathrm{d} \omega\right\rangle \\
& =\int_{-\infty}^{\infty}\left\langle\frac{B(-\mathrm{i} \omega)^{*} h}{z-\mathrm{i} \omega}, x(\mathrm{i} \omega)\right\rangle \mathrm{d} \omega \\
& =\int_{-\infty}^{\infty}\left\langle\frac{B(\mathrm{i} \omega)^{*} h}{z+\mathrm{i} \omega},(J x)(\mathrm{i} \omega)\right\rangle \mathrm{d} \omega \\
& =\frac{1}{\sqrt{\operatorname{Re} z}} \int_{-\infty}^{\infty}\left\langle J B(\mathrm{i} \omega)^{*} \frac{\sqrt{\operatorname{Re} z}}{z+\mathrm{i} \omega} h, x(\mathrm{i} \omega)\right\rangle \mathrm{d} \omega \\
& =\frac{1}{\sqrt{\operatorname{Re} z}}\left\langle\left(\Gamma_{B}\right)^{*} f_{z} h, x\right\rangle
\end{aligned}
$$

and so

$$
\left\|C(z I-A)^{-1}\right\| \leqslant m \frac{1}{\sqrt{\operatorname{Re} z}}
$$

by part (iii) of Theorem 2.1.

(iv) Let $\phi \in H^{\prime}$. Then part (iii) implies

$$
\left\|\phi C(z I-A)^{-1}\right\| \leqslant \frac{m}{\sqrt{\operatorname{Re} z}}\|\phi\| \quad \text { for every } z \in \mathbb{C}_{+} .
$$

Using the fact that $A$ generates a contraction semigroup, the main result of Jacob and Partington [8] shows that $\phi C$ is an admissible observation operator for $T(t)$.

Acknowledgements. We thank S. Treil for fruitful discussions. B.J. and S.P. gratefully acknowledge support by EPSRC.

While this paper was being refereed, O. Blasco pointed out to us that the inequality of $\operatorname{BMO}(H)$ and $\operatorname{WBMO}(H)$ in Lemma 2.2 can also be deduced from Corollaries 1 and 2 in $[\mathbf{1}]$.

\section{References}

1. O. BlAsCO, Operators from $H^{1}$ into a Banach space and vector valued measures, Geometric aspects of Banach spaces, London Mathematical Society Lecture Note Series, vol. 140, pp. 87-93 (Cambridge University Press, 1989).

2. O. Blasco, Vector-valued analytic functions of bounded mean oscillation, Illinois $\mathrm{J}$. Math. 41 (1997), 532-558.

3. F. F. Bonsall, Boundedness of Hankel matrices, J. Lond. Math. Soc. 29 (1984), 289-300.

4. J. B. Garnett, Bounded analytic functions (Academic, 1981).

5. T. A. Gillespie, S. Pott, S. Treil and A. Volberg, Logarithmic growth for weighted Hilbert transforms and vector Hankel operators, submitted (2001).

6. S. Hansen And G. Weiss, The operator Carleson measure criterion for admissibility of control operators for diagonal semigroups on $\ell_{2}$, Syst. Contr. Lett. 16 (1991), 219-227. 
7. S. HANSEN AND G. Weiss, New results on the operator Carleson measure criterion. IMA J. Math. Contr. Informat. 14 (1997), 3-32.

8. B. JaCOB And J. R. PARTington, The Weiss conjecture on admissibility of observation operators for contraction semigroups, Integ. Eqns Operat. Theory 40 (2001), 231-243.

9. B. JACOB AND H. ZWART, Disproof of two conjectures of George Weiss, submitted (2000).

10. F. Nazarov, S. Treil and A. Volberg, Counterexample to the infinite dimensional Carleson Embedding Theorem, C. R. Acad. Sci. Paris Sér. I 325 (1997), 383-388.

11. J. R. Partington And G. Weiss, Admissible observation operators for the right shift semigroup, Math. Contr. Sign. Syst. 13 (2000), 179-192.

12. G. WeISs, Admissible observation operators for linear semigroups, Israel J. Math. 65 (1989), 17-43.

13. G. WeIss, Two conjectures on the admissibility of control operators, in Estimation and Control of Distributed Parameter Systems (ed. F. Kappel and W. Desch), pp. 367-378 (Birkhäuser, 1991).

14. G. WeISS, A powerful generalization of the Carleson measure theorem?, in Open problems in mathematical systems theory and control (ed. V. Blondel, E. Sontag, M. Vidyasagar and J. Willems) (Springer, 1998).

15. H. Zwart, B. JaCOB And O. Staffans, Weak admissibility does not imply admissibility for analytic semigroups, submitted (2000). 